\title{
Adverse effects of remdesivir, hydroxychloroquine, and lopinavir/ritonavir when used for COVID-19: systematic review and meta-analysis of randomized trials
}

\author{
Ariel Izcovich, ${ }^{1}$ Reed AC Siemieniuk, ${ }^{2}$ Jessica J Bartoszko, ${ }^{2}$ Long Ge, ${ }^{3}$ Dena Zeraatkar, ${ }^{2}$ Elena \\ $\mathrm{Kum}^{2}$, Assem M. Khamis, ${ }^{4}$ Bram Rochwerg, ${ }^{2}$ Thomas Agoritsas, ${ }^{2,5}$ Derek K Chu, ${ }^{2,6}$ Shelley L \\ McLeod, ${ }^{7,8}$ Reem A Mustafa, ${ }^{2,9}$ Per O Vandvik, ${ }^{10}$ Romina Brignardello-Petersen ${ }^{2}$
}

\author{
1 Servicio de Clinica Médica del Hospital Alemán, Buenos Aires, Argentina \\ 2 Department of Health Research Methods, Evidence, and Impact, McMaster University, 1280 Main St W, \\ Hamilton, ON L8S 4 L8, Canada \\ 3 Evidence Based Social Science Research Center, School of Public Health, Lanzhou University, Lanzhou, Gansu, \\ China \\ 4 Hull York Medical School, University of Hull \\ 5 Division of General Internal Medicine \& Division of Clinical Epidemiology, University Hospitals of Geneva, \\ Geneva, Switzerland \\ 6 Department of Medicine, McMaster University, Hamilton, ON, Canada \\ 7 Schwartz/Reisman Emergency Medicine Institute, Sinai Health, Toronto, ON, Canada \\ 8 Department of Family and Community Medicine, University of Toronto, Toronto, ON, Canada \\ 9 Department of Medicine, University of Kansas Medical Center, Kansas City, MO, USA \\ 10 MAGIC Evidence Ecosystem Foundation, Oslo, Norway
}

Word count: 3458

Abstract word count: 400

Figures: 1

Tables:2

Supplementary files:2 


\begin{abstract}
Introduction: In an attempt to improve outcomes for patients with coronavirus disease 19 (COVID-19), several drugs, such as remdesivir, hydroxychloroquine (with or without azithromycin), and lopinavir/ritonavir, have been evaluated for treatment. While much attention focuses on potential benefits of these drugs, this must be weighed against their adverse effects.
\end{abstract} Methods: We searched 32 databases in multiple languages from 1 December 2019 to 27 October 2020. We included randomized trials if they compared any of the drugs of interest to placebo or standard care, or against each other. A related world health organization (WHO) guideline panel selected the interventions to address and identified possible adverse effects that might be important to patients. Pairs of reviewers independently extracted data and assessed risk of bias. We analyzed data using a fixed-effects pairwise meta-analysis and assessed the certainty of evidence using the GRADE approach.

Results: We included 16 randomized trials which enrolled 8226 patients. Compared to standard care or placebo, low certainty evidence suggests that remdesivir may not have an important effect on acute kidney injury (risk difference [RD] 8 fewer per 1000, 95\% confidence interval (CI): 27 fewer to 21 more) or cognitive dysfunction/delirium (RD 3 more per 1000, 95\% CI: 12 fewer to 19 more). Low certainty evidence suggests that hydroxychloroquine may increase the risk of serious cardiac toxicity (RD 10 more per 1000, 95\% CI: 0 more to 30 more) and cognitive dysfunction/delirium (RD 33 more per 1000, 95\% CI: 18 fewer to 84 more), whereas moderate certainty evidence suggests hydroxychloroquine probably increases the risk of diarrhoea (RD 106 more per 1000, 95\% CI: 48 more to 175 more) and nausea and/or vomiting (RD 62 more per 1000, 95\% CI: 23 more to 110 more) compared to standard care or placebo. Low certainty evidence suggests lopinavir/ritonavir may increase the risk of diarrhoea (RD 168 more per 1000, 95\% CI: 58 more to 330 more) and nausea and/or vomiting (RD 160 more per 1000, 95\% CI: 100 more to 210 more) compared to standard care or placebo.

Conclusion: Hydroxychloroquine probably increases the risk of diarrhoea and nausea and/or vomiting and may increase the risk of cardiac toxicity and cognitive dysfunction/delirium. Remdesivir may have no effect on risk of acute kidney injury or cognitive dysfunction/delirium. Lopinavir/ritonavir may increase the risk of diarrhoea and nausea and/or vomiting. These findings provide important information to support the development of evidence-based management strategies for patients with COVID-19. 


\section{Introduction}

As of November 16, 2020, there are 54.6 million cumulative cases of COVID-19 worldwide, and at least 1.3 million deaths. ${ }^{1}$ Several drugs have been used for the treatment of patients with COVID-19, often without high quality evidence demonstrating efficacy. Three drugs that have been used for COVID-19 include remdesivir, hydroxychloroquine with or without azithromycin, and lopinavir/ritonavir. None of these drugs have high certainty evidence evaluating their effectiveness for key patient-important outcomes such as mortality, need for mechanical ventilation, duration of hospital stay or time to clinical improvement. ${ }^{2}$

We are conducting a living systematic review and network meta-analysis to provide a summary of the evidence for all drugs used in the treatment of COVID-19. ${ }^{2}$ Until now, we have not found that any one of these drugs increases the risk of adverse effects leading to drug continuation when compared to standard care or another drug treatment. However, we have not evaluated drug-specific adverse effects, which patients might consider to be important when making decisions about whether to use or not use a drug, particularly in the face of considerable uncertainty regarding their desirable effects.

Building on the work of the living systematic review, the aim of this paper is to summarize the best available evidence addressing drug-specific adverse effects in COVID-19. This evidence synthesis is part of the BMJ-Rapid Recommendations project, ${ }^{3}$ to inform World Health Organization (WHO) Living Guidelines on drugs for treatment of COVID-19. ${ }^{4,5}$

\section{Methods}

We followed the preferred reporting items for systematic reviews and meta-analyses (PRISMA) guidelines for reporting. ${ }^{6}$

\section{Eligibility criteria}

As selected by the linked guideline panel we included randomised clinical trials (RCTs) that included people with suspected, probable, or confirmed COVID-19 comparing remdesivir, hydroxychloroquine, and lopinavir/ritonavir, alone or in combination with other drugs, for 
treatment against one another or against no intervention, placebo, or standard care, and reported on drug-specific adverse events of interest (see outcome identification below). We included trials regardless of publication status (peer reviewed, in press, or preprint) or language. No restrictions were applied based on severity of COVID-19 illness or setting in which the trial was conducted (outpatient, hospital, ICU, etc). We excluded studies in which remdesivir, hydroxychloroquine, and lopinavir/ritonavir were used for prophylaxis and studies in which different doses of the same intervention were compared.

\section{Information sources}

We performed daily searches from Monday to Friday using the WHO COVID-19 database for eligible studies, which is a comprehensive multilingual source of global literature on COVID19. ${ }^{7}$ Prior to its merge with the WHO COVID-19 database on 9 October 2020, we also performed daily searches for eligible studies from Monday to Friday in the US Centers for Disease Control and Prevention (CDC) COVID-19 Research Articles Downloadable Database. ${ }^{8}$ To identify RCTs, we filtered the results from the CDC's database through a validated and highly sensitive machine learning model. ${ }^{9}$ In addition, we searched six Chinese databases. We adapted the search terms for COVID-19 developed by the CDC to the Chinese language. For the Chinese literature search, we also included search terms for randomised trials.

We also used living evidence retrieval services to identify any trials that might have been missed with traditional search methods. These included the Living Overview of the Evidence (L-OVE) COVID-19 Repository by the Epistemonikos Foundation ${ }^{10}$ and the Systematic and Living Map on COVID-19 Evidence by the Norwegian Institute of Public Health, in collaboration with the Cochrane Canada Centre at McMaster University. ${ }^{11}$ We searched all English information sources from 1 December 2019 to 27 October 2020, and the Chinese literature from inception of the databases to 16 October 2020. A complete list of information sources is available in supplementary text 1 .

\section{Study selection}


Using systematic review software, Covidence, ${ }^{12}$ following training and calibration exercises, pairs of reviewers independently screened all titles and abstracts, followed by full texts of trials that were identified as potentially eligible. A third reviewer adjudicated conflicts.

\section{Data collection}

For each eligible trial, pairs of reviewers extracted data independently using a standardised, pilot-tested data extraction form. Reviewers collected information on trial characteristics (trial registration, publication status, study status, design), participant characteristics (country, age, sex, smoking habits, comorbidities), and outcomes of interest. Reviewers resolved discrepancies by discussion and, when necessary, with adjudication by a third party.

\section{Outcome identification}

A linked WHO-BMJ Rapid Recommendations guideline panel ${ }^{4}$ consisting of patients, clinicians, and research methodologists with representation from all WHO geographic regions provided input on potentially important adverse effects of the medications. If any of the panelists believed a specific adverse effect was possible and might influence the decision to use or not use each drug, it was included in this systematic review as an outcome of interest. Panelists were asked to focus on adverse effects important to patients, rather than surrogate measures. For example, we considered clinically important cardiac toxicity including arrhythmias important, but did not consider changes to the QT interval important.

The panel identified specific adverse effects for each drug. For remdesivir, we included acute kidney injury. For hydroxychloroquine and hydroxychloroquine with azithromycin, we included cardiac toxicity, diarrhoea, and nausea and/or vomiting. For lopinavir/ritonavir, we included acute kidney injury, diarrhoea, and nausea and/or vomiting. For all of the drugs, we included cognitive dysfunction/delirium and fatigue. We included studies in which researchers used any definitions of these outcomes. In cases in which the definitions did appropriately reflect what is important to patients, we rated down the certainty of the evidence for indirectness (see certainty of the evidence below).

\section{Risk of bias within individual studies}


For each eligible trial and outcome, following training and calibration exercises, reviewers used a revision of the Cochrane tool for assessing risk of bias in RCTs (RoB 2.0) ${ }^{13}$ to rate trials as either at i) low risk of bias, ii) some concerns—-probably low risk of bias, iii) some concernsprobably high risk of bias, or iv) high risk of bias, across the following domains: bias arising from the randomisation process; bias due to departures from the intended intervention; bias due to missing outcome data; bias in measurement of the outcome; bias in selection of the reported results, including deviations from the registered protocol; and bias arising from early termination for benefit. We rated trials at high risk of bias overall if one or more domains were rated as "some concerns_-probably high risk of bias" or as "high risk of bias", and as low risk of bias overall if all domains were rated as "some concerns - probably low risk of bias" or "low risk of bias". Reviewers resolved discrepancies by discussion and, when not possible, with adjudication by a third party.

\section{Data synthesis}

\section{Measures of effect and statistical analysis}

We summarised the effect of interventions on dichotomous outcomes using odds ratios (ORs) and corresponding 95\% confidence intervals (CIs). We conducted frequentist fixed-effects pairwise meta-analyses using the R package "meta" in RStudio Version 1.3.1093. ${ }^{14}$ We used fixed rather than random effects for the primary analysis because for many of the interventions, the evidence consisted of two or fewer trials or there were several studies with few events. For outcomes in which there were more than one trial with no events in both groups, we metaanalysed the data using risk differences $(\mathrm{RD})$ rather than odds ratios.

\section{Certainty of the evidence}

We assessed the certainty of evidence using the grading of recommendations assessment, development and evaluation (GRADE) approach. ${ }^{15}$ Two methodologists with experience in using GRADE rated each domain for each comparison separately and resolved discrepancies by consensus. We rated the certainty for each comparison and outcome as high, moderate, low, or very low, based on considerations of risk of bias, inconsistency, indirectness, publication bias, and imprecision. We made judgments of imprecision using a minimally contextualised approach with the null effect as a threshold. This minimally contextualised approach considers whether the 
CI includes the null effect, or, when the point estimate is close to the null effect, whether the CI lies within the boundaries of small but important benefit and harm. ${ }^{16}$ Additionally we analysed if the total number of patients included in the meta-analysis was less than the required number of patients generated by a conventional sample size calculation for a single adequately powered trial to define if optimal information size (OIS) was met. For some of the interventions, extensively implemented in other clinical scenarios, we used indirect evidence to complement the certainty of evidence judgments. We created GRADE evidence summaries (Summary of Findings tables) using the MAGIC Authoring and Publication Platform (www.magicapp.org) to provide user friendly formats for clinicians and patients and to allow re-use in the context of clinical practice guidelines for COVID-19. ${ }^{4,5}$ We calculated the absolute risks and risk differences from the ORs (and their CIs) and the mean risk in the control groups across all of the included trials. In cases where no events were reported in the control arm of any of the included studies, we calculated baseline risks based on other comparisons for the same outcome.

\section{Subgroup and sensitivity analyses}

We performed Bayesian random-effects meta-analysis using the bayesmeta package. ${ }^{17}$ We used a plausible prior for the variance parameter and a uniform prior for the effect parameter, as suggested in an empirical study using pre-specified empiric priors as a sensitivity analysis for all comparisons. ${ }^{18}$ We did not conduct any subgroup analyses.

\section{Results}

\section{Study identification}

After screening 14,806 titles and abstracts and 300 full texts, we included sixteen unique RCTs with 8,152 patients that informed on drug-specific adverse events (figure 1). ${ }^{19-34}$ We did not identify any additional eligible RCTs through the living evidence retrieval services. Two studies reported adverse effects for remdesivir, ${ }^{19,33}$ ten for hydroxychloroquine, ${ }^{21-26,29-32}$ one for hydroxychloroquine plus azithromycin, ${ }^{21}$ and four for lopinavir/ritonavir. ${ }^{20,27,28,34}$ Of the sixteen eligible RCTs, 13 have been published in peer reviewed journals, and 3 only as preprints. ${ }^{22,24,26}$ All of the trials were registered, published in English and most evaluated treatment in patients admitted to hospital with COVID-19 (15/16; 93.7\%). Most of the trials were conducted in China $(10 / 16 ; 62.5 \%)$. Table 1 presents the characteristics of the included studies. Additional study 
medRxiv preprint doi: https://doi.org/10.1101/2020.11.16.20232876; this version posted November 18, 2020. The copyright holder for this preprint (which was not certified by peer review) is the author/funder, who has granted medRxiv a license to display the preprint in perpetuity.

It is made available under a CC-BY-NC-ND 4.0 International license .

characteristics, outcome data, and risk of bias assessments for each study are available in the supplementary file.

Figure 1. Study selection (PRISMA form LNMA - Jess can help. The bottom cell should have number of trials for each drug separately.) 
medRxiv preprint doi: https://doi.org/10.1101/2020.11.16.20232876; this version posted November 18, 2020. The copyright holder for this preprint (which was not certified by peer review) is the author/funder, who has granted medRxiv a license to display the preprint in perpetuity.

It is made available under a CC-BY-NC-ND 4.0 International license .

\section{As of 27 October 2020,}

\section{0 records identified from literature search}

14607 English bibliographic databases and pre-print servers

523 Chinese bibliographic databases and pre-print servers

20 records identified from external sources

19 Epistemonikos COVID-19 Evidence

14506 records excluded for not being relevant

\section{6 records after duplicates removed}

\section{0 full text articles assessed for eligibility}

118 randomized trials $+\mathbf{2}$ meta-analyses of randomized trials included

\begin{tabular}{|l|l|}
\hline $\begin{array}{l}12 \text { preprints of published trials } \\
1 \text { correction } \\
1 \text { preliminary report of published final report }\end{array}$ & $\begin{array}{l}10 \text { duplicates } \\
2 \text { post-hoc / }\end{array}$ \\
\cline { 2 - 3 } & $\mathbf{9 4}$ publications with $\mathbf{1 0 6}$ unique randomized trials (data from 17 unpublished randomized trials in 2 m \\
89 English and 5 Chinese texts \\
64 published and 30 preprints \\
\hline $\mathbf{8 5}$ randomised trials included in this systematic review \\
$\mathbf{1 6}$ randomised trials reported on drug-specific adverse events \\
$\mathbf{1 0}$ on hydroxychloroquine \\
$\mathbf{4}$ on lopinavir/ritonavir \\
$\mathbf{2}$ on remdesivir
\end{tabular}


medRxiv preprint doi: https://doi.org/10.1101/2020.11.16.20232876; this version posted November 18, 2020. The copyright holder for this preprint (which was not certified by peer review) is the author/funder, who has granted medRxiv a license to display the preprint in perpetuity.

It is made available under a CC-BY-NC-ND 4.0 International license .

Table 1. Characteristics of the included trials.

\begin{tabular}{|c|c|c|c|c|c|c|c|c|c|c|}
\hline Study & $\begin{array}{l}\text { Publication } \\
\text { status, } \\
\text { registration } \\
\text { No }\end{array}$ & $\begin{array}{l}\text { No of } \\
\text { participants }\end{array}$ & Country & $\begin{array}{l}\text { Mean } \\
\text { age } \\
\text { (years) }\end{array}$ & $\begin{array}{l}\text { Men } \\
(\%)\end{array}$ & $\begin{array}{l}\text { Type of care, } \\
\text { comorbidities }\end{array}$ & $\begin{array}{l}\text { Severity } \\
\text { (accordin } \\
\text { g to study } \\
\text { authors) }\end{array}$ & $\begin{array}{l}\text { Mechanica } \\
\text { l } \\
\text { ventilation } \\
\text { at baseline } \\
(\%)\end{array}$ & $\begin{array}{l}\text { Treatments (dose } \\
\text { and duration) }\end{array}$ & Outcomes \\
\hline $\begin{array}{l}\text { Beigel } \\
2020 ; \\
\text { ACTT-1 }^{19}\end{array}$ & $\begin{array}{l}\text { Published, } \\
\text { NCT0428070 } \\
5\end{array}$ & 1062 & $\begin{array}{l}\text { USA, } \\
\text { Denmark } \\
\text {, UK, } \\
\text { Greece, } \\
\text { Germany } \\
\text {, Korea, } \\
\text { Mexico, } \\
\text { Spain, } \\
\text { Japan, } \\
\text { Singapor } \\
\text { e }\end{array}$ & 58.9 & 64.4 & $\begin{array}{l}\text { Inpatient; coronary } \\
\text { artery disease } \\
(11.9 \%) \text {; congestive } \\
\text { heart failure }(5.6 \%) \text {; } \\
\text { diabetes }(30.6 \%) ; \\
\text { hypertension } \\
(50.7 \%) \text {; asthma } \\
(11.4 \%) \text {; chronic } \\
\text { oxygen requirement } \\
(2.2 \%) \text {; chronic } \\
\text { respiratory disease } \\
(7.6 \%)\end{array}$ & $\begin{array}{l}\text { Severe } \\
(90.1 \%)\end{array}$ & 45.0 & $\begin{array}{l}\text { Remdesivir ( } 200 \\
\mathrm{mg} / \text { day for } 1 \text { day, } \\
\text { then } 100 \mathrm{mg} / \text { day for } \\
9 \text { days); placebo }\end{array}$ & $\begin{array}{l}\text { Acute kidney injury; } \\
\text { Cognitive } \\
\text { dysfunction/delirium }\end{array}$ \\
\hline $\begin{array}{l}\text { Cao 2020; } \\
\text { LOTUS }_{\text {China }^{20}}\end{array}$ & $\begin{array}{l}\text { Published, } \\
\text { ChiCTR2000 } \\
029308\end{array}$ & 199 & China & 58.0 & 60.3 & $\begin{array}{l}\text { Inpatient; } \\
\text { cerebrovascular } \\
\text { disease }(6.5 \%) ; \\
\text { diabetes }(11.6 \%)\end{array}$ & $\begin{array}{l}\text { Severe } \\
(100 \%)\end{array}$ & 16.1 & $\begin{array}{l}\text { Lopinavir-ritonavir } \\
(400 \mathrm{mg} \text { and } 100 \\
\mathrm{mg} \text { twice daily for } \\
14 \text { days); standard } \\
\text { care }\end{array}$ & $\begin{array}{l}\text { Acute kidney injury; } \\
\text { Diarrhoea; Nausea } \\
\text { and/or vomiting; } \\
\text { Fatigue }\end{array}$ \\
\hline $\begin{array}{l}\text { Cavalcanti, } \\
2020^{21}\end{array}$ & $\begin{array}{l}\text { Published, } \\
\text { NCT0432212 } \\
3\end{array}$ & 667 & Brazil & 50.3 & 58.4 & $\begin{array}{l}\text { Inpatient; intensive } \\
\text { care }(13.8 \%) ; \text { heart } \\
\text { failure }(1.5 \%) ; \\
\text { diabetes }(19.1 \%) ; \\
\text { hypertension } \\
(38.3 \%) \text {; asthma } \\
(6.0 \%) \text {; chronic } \\
\text { obstructive } \\
\text { pulmonary disease } \\
(1.8 \%)\end{array}$ & $\begin{array}{l}\text { Mild/Mod } \\
\text { erate } \\
(100 \%)\end{array}$ & 0 & $\begin{array}{l}\text { Hydroxychloroquin } \\
\text { e ( } 400 \text { mg twice } \\
\text { daily for } 7 \text { days); } \\
\text { hydroxychloroquine } \\
\text { (400 mg twice daily } \\
\text { for } 7 \text { days), } \\
\text { azithromycin (500 } \\
\text { mg/day for } 7 \text { days); } \\
\text { standard care }\end{array}$ & $\begin{array}{l}\text { Cardiac toxicity; } \\
\text { Nausea and/or } \\
\text { vomiting }\end{array}$ \\
\hline Chen $2020^{22}$ & $\begin{array}{l}\text { Preprint, } \\
\text { ChiCTR2000 } \\
029559\end{array}$ & 62 & China & 44.7 & 46.8 & Inpatient; NR & $\begin{array}{l}\text { Mild/mod } \\
\text { erate } \\
(100 \%)\end{array}$ & NR & $\begin{array}{l}\text { Hydroxychloroquin } \\
\text { e ( } 200 \text { mg twice } \\
\text { daily for } 5 \text { days); } \\
\text { standard care }\end{array}$ & Cardiac toxicity \\
\hline Chen $2020^{23}$ & $\begin{array}{l}\text { Published, } \\
\text { NCT0426151 } \\
7\end{array}$ & 30 & China & 48.6 & 70.0 & $\begin{array}{l}\text { Inpatient; diabetes } \\
(6.7 \%) \text {; hypertension } \\
(26.7 \%) \text {; chronic } \\
\text { obstructive } \\
\text { pulmonary disease } \\
(3.3 \%)\end{array}$ & $\begin{array}{l}\text { Mild/mod } \\
\text { erate } \\
(100 \%)\end{array}$ & NR & $\begin{array}{l}\text { Hydroxychloroquin } \\
\text { e ( } 400 \mathrm{mg} / \text { day for } 5 \\
\text { days); standard care }\end{array}$ & $\begin{array}{l}\text { Diarrhoea; Nausea } \\
\text { Nomiting }\end{array}$ \\
\hline Chen $2020^{24}$ & $\begin{array}{l}\text { Preprint, } \\
\text { ChiCTR2000 } \\
030054\end{array}$ & 48 & China & 46.9 & 45.8 & $\begin{array}{l}\text { Inpatient; diabetes } \\
(18.8 \%) \text {; } \\
\text { hypertension } \\
(16.7 \%)\end{array}$ & $\begin{array}{l}\text { Mild/mod } \\
\text { erate } \\
(100 \%)\end{array}$ & NR & $\begin{array}{l}\text { Chloroquine } \\
\text { ( } 1000 / \text { day for } 1 \text { day, } \\
\text { then } 500 \mathrm{mg} / \text { day for } \\
9 \text { days); } \\
\text { hydroxychloroquine } \\
\text { ( } 200 \mathrm{mg} \text { twice daily } \\
\text { for } 10 \text { days); } \\
\text { standard care }\end{array}$ & $\begin{array}{l}\text { Cardiac toxicity; } \\
\text { Diarrhoea; Nausea } \\
\text { and/or vomiting }\end{array}$ \\
\hline Chen $2020^{25}$ & $\begin{array}{l}\text { Preprint, } \\
\text { NCT0438438 } \\
0\end{array}$ & 33 & Taiwan & 32.9 & 57.6 & Inpatient & $\begin{array}{l}\text { Mild/Mod } \\
\text { erate } \\
(100 \%)\end{array}$ & 0 & $\begin{array}{l}\text { Hydroxychloroquin } \\
\text { e ( } 400 \text { mg twice } \\
\text { daily for } 1 \text { day, then } \\
200 \text { mg twice daily } \\
\text { for } 6 \text { days); standard } \\
\text { care }\end{array}$ & $\begin{array}{l}\text { Diarrhoea; Nausea } \\
\text { and/or vomiting }\end{array}$ \\
\hline $\begin{array}{l}\text { Horby 2020; } \\
\text { RECOVER } \\
\mathrm{Y}^{26}\end{array}$ & $\begin{array}{l}\text { Published, } \\
\text { NCT0438193 } \\
6\end{array}$ & 4716 & UK & 65.3 & 62.2 & $\begin{array}{l}\text { Inpatient; heart } \\
\text { disease }(25.7 \%) \text {; } \\
\text { diabetes }(27.2 \%) \text {; } \\
\text { chronic lung disease } \\
(22.2 \%) \text {; } \\
\text { tuberculosis }(0.3 \%)\end{array}$ & NR & 16.8 & $\begin{array}{l}\text { Hydroxychloroquin } \\
\text { e ( } 800 \text { mg at zero } \\
\text { and } 6 \text { hours, then } \\
400 \mathrm{mg} \text { twice daily } \\
\text { for } 9 \text { days); standard } \\
\text { care }\end{array}$ & Cardiac toxicity \\
\hline $\begin{array}{l}\text { Huang } \\
2020^{27}\end{array}$ & $\begin{array}{l}\text { Published, } \\
\text { ChiCTR2000 } \\
029387\end{array}$ & 101 & China & 42.5 & 45.5 & Inpatient & $\begin{array}{l}\text { Mild/mod } \\
\text { erate } \\
(100 \%)\end{array}$ & NR & $\begin{array}{l}\text { Ribavirin ( } 400-600 \\
\text { mg three times daily } \\
\text { for } 14 \text { days), } \\
\text { interferon-alfa ( } 5 \\
\text { mg twice daily for } \\
14 \text { days); lopinavir- } \\
\text { ritonavir ( } 400 \mathrm{mg} \\
\text { and } 100 \mathrm{mg} \text { twice } \\
\text { daily for } 14 \text { days), } \\
\text { interferon-alfa ( } 5 \\
\text { mg twice daily for } \\
14 \text { days); ribavirin } \\
\text { ( } 400-600 \mathrm{mg} \text { three } \\
\text { times daily for } 14 \\
\text { days), lopinavir- } \\
\text { ritonavir ( } 400 \mathrm{mg} \\
\text { and } 100 \mathrm{mg} \text { twice } \\
\text { daily for } 14 \text { days), } \\
\text { interferon-alfa (5 } \\
\text { mg twice daily for }\end{array}$ & $\begin{array}{l}\text { Acute Kidney injury; } \\
\text { Diarrhoea; Nausea } \\
\text { and/or vomiting }\end{array}$ \\
\hline
\end{tabular}


medRxiv preprint doi: https://doi.org/10.1101/2020.11.16.20232876; this version posted November 18,2020 . The copyright holder for this preprint (which was not certified by peer review) is the author/funder, who has granted medRxiv a license to display the preprint in perpetuity.

It is made available under a CC-BY-NC-ND 4.0 International license .

\begin{tabular}{|c|c|c|c|c|c|c|c|c|c|c|}
\hline $\begin{array}{l}\mathrm{Li} 2020 ; \\
\text { ELACOI }^{28}\end{array}$ & $\begin{array}{l}\text { Published, } \\
\text { NCT0425288 } \\
5\end{array}$ & 86 & China & 49.4 & 46.5 & $\begin{array}{l}\text { Inpatient; } \\
\text { cardiovascular } \\
\text { disease }(2.3 \%) \text {; } \\
\text { diabetes }(2.3 \%) \text {; } \\
\text { hypertension } \\
(10.5 \%)\end{array}$ & $\begin{array}{l}\text { Mild/mod } \\
\text { erate } \\
(100 \%)\end{array}$ & 0 & $\begin{array}{l}14 \text { days) } \\
\text { Lopinavir-ritonavir } \\
\text { ( } 400 \text { mg and } 100 \\
\text { mg twice daily for } 7 \\
\text { to } 14 \text { days); } \\
\text { umifenovir ( } 200 \mathrm{mg} \\
\text { three times daily for } \\
7 \text { to } 14 \text { days); } \\
\text { standard care }\end{array}$ & $\begin{array}{l}\text { Diarrhoea; Nausea } \\
\text { and/or vomiting }\end{array}$ \\
\hline $\begin{array}{l}\text { Lyngbakken } \\
2020^{29}\end{array}$ & $\begin{array}{l}\text { Published, } \\
\text { NCT0431637 } \\
7\end{array}$ & 53 & Norway & 62.0 & 66.0 & $\begin{array}{l}\text { Inpatient; coronary } \\
\text { heart disease }(9.4 \%) \text {; } \\
\text { diabetes }(17.0 \%) ; \\
\text { hypertension } \\
(32.1 \%) \text {; chronic } \\
\text { obstructive } \\
\text { pulmonary disease } \\
\text { or asthma }(26.4 \%)\end{array}$ & $\begin{array}{l}\text { Mild } / \bmod \\
\text { erate }(0 \%)\end{array}$ & 0 & $\begin{array}{l}\text { Hydroxychloroquin } \\
\text { e ( } 400 \text { mg twice } \\
\text { daily for } 7 \text { days); } \\
\text { standard care }\end{array}$ & $\begin{array}{l}\text { Diarrhoea; Nausea } \\
\text { and/or vomiting }\end{array}$ \\
\hline $\begin{array}{l}\text { Skipper } \\
2020^{30}\end{array}$ & $\begin{array}{l}\text { Published, } \\
\text { NCT0430866 } \\
8\end{array}$ & 491 & $\begin{array}{l}\text { USA, } \\
\text { Canada }\end{array}$ & 40.0 & 45.8 & $\begin{array}{l}\text { Outpatient; } \\
\text { cardiovascular } \\
\text { disease }(1.2 \%) ; \\
\text { diabetes }(3.9 \%) ; \\
\text { hypertension } \\
(11.0 \%) ; \text { asthma } \\
(10.4 \%) ; \text { chronic } \\
\text { lung disease }(0.4 \%)\end{array}$ & $\begin{array}{l}\text { Mild/mod } \\
\text { erate } \\
(100 \%)\end{array}$ & 0 & $\begin{array}{l}\text { Hydroxychloroquin } \\
\text { e ( } 800 \mathrm{mg} \text { at zero } \\
\text { hours, then } 600 \mathrm{mg} \\
6 \text { to } 8 \text { hours later, } \\
\text { then } 600 \mathrm{mg} / \text { day for } \\
4 \text { days); placebo }\end{array}$ & $\begin{array}{l}\text { Cardiac toxicity; } \\
\text { Diarrhoea; Nausea } \\
\text { Nomiting; Cognitive } \\
\text { dysfunction/delirium }\end{array}$ \\
\hline Tang $2020^{31}$ & $\begin{array}{l}\text { Published, } \\
\text { ChiCTR2000 } \\
029868\end{array}$ & 150 & China & 46.1 & 55.0 & $\begin{array}{l}\text { Inpatient; diabetes } \\
(14.0 \%) \text {; } \\
\text { hypertension }(6.0 \%)\end{array}$ & $\begin{array}{l}\text { Mild/mod } \\
\text { erate } \\
(99.0 \%) \\
\text { severe } \\
(1.0 \%)\end{array}$ & NR & $\begin{array}{l}\text { Hydroxychloroquin } \\
\text { e ( } 1200 \mathrm{mg} / \text { day for } \\
3 \text { days, then } 800 \\
\text { mg/day until } 14 \text { to } \\
21 \text { days of total } \\
\text { treatment); standard } \\
\text { care }\end{array}$ & $\begin{array}{l}\text { Cardiac toxicity; } \\
\text { Diarrhoea; Nausea } \\
\text { Nomiting; Cognitive }\end{array}$ \\
\hline $\begin{array}{l}\text { Ulrich 2020; } \\
\text { TEACH }^{32}\end{array}$ & $\begin{array}{l}\text { Published, } \\
\text { NCT0436974 } \\
2\end{array}$ & 128 & USA & 66.2 & 59.4 & $\begin{array}{l}\text { Inpatient; non- } \\
\text { hypertensive } \\
\text { cardiovascular } \\
\text { disease }(25.6 \%) ; \\
\text { diabetes }(32.0 \%) \text {; } \\
\text { hypertension } \\
(57.8 \%) \text {; asthma } \\
(15.6 \%) \text {; chronic } \\
\text { obstructive } \\
\text { pulmonary disease } \\
(7.0 \%)\end{array}$ & $\begin{array}{l}\text { Mild } / \bmod \\
\text { erate }(0 \%)\end{array}$ & 0.78 & $\begin{array}{l}\text { Hydroxychloroquin } \\
\text { e ( } 400 \mathrm{mg} \text { twice } \\
\text { daily for } 1 \text { day, then } \\
200 \mathrm{mg} \text { twice daily } \\
\text { for } 4 \text { days); placebo }\end{array}$ & Cardiac toxicity \\
\hline $\begin{array}{l}\text { Wang } \\
2020^{33}\end{array}$ & $\begin{array}{l}\text { Published, } \\
\text { NCT0425765 } \\
6\end{array}$ & 237 & China & 65.0 & 59.3 & $\begin{array}{l}\text { Inpatient; } \\
\text { cardiovascular } \\
\text { disease }(7.2 \%) ; \\
\text { diabetes }(23.7 \%) \text {; } \\
\text { hypertension } \\
(43.2 \%)\end{array}$ & $\begin{array}{l}\text { Severe } \\
(100 \%)\end{array}$ & 16.1 & $\begin{array}{l}\text { Remdesivir ( } 200 \\
\mathrm{mg} / \text { day for } 1 \text { day, } \\
\text { then } 100 \mathrm{mg} / \text { day for } \\
9 \text { days); placebo }\end{array}$ & Acute kidney injury \\
\hline $\begin{array}{l}\text { Zheng } \\
2020^{34}\end{array}$ & $\begin{array}{l}\text { Published, } \\
\text { ChiCTR2000 } \\
029496\end{array}$ & 89 & China & 46.7 & 47.2 & $\begin{array}{l}\text { Inpatient; chronic } \\
\text { bronchitis }(2.0 \%)\end{array}$ & $\begin{array}{l}\text { Mild/mod } \\
\text { erate } \\
(94.4 \%) \\
\text { severe } \\
(5.6 \%)\end{array}$ & NR & $\begin{array}{l}\text { Novaferon ( } 20 \mu \mathrm{g} \\
\text { twice daily for } 7 \text { to } \\
10 \text { days); novaferon, } \\
\text { lopinavir-ritonavir } \\
\text { ( } 400 \text { mg and } 100 \\
\text { mg twice daily for } 7 \\
\text { to } 10 \text { days); } \\
\text { lopinavir-ritonavir } \\
\text { ( } 400 \text { mg and } 100 \\
\text { mg twice daily for } 7 \\
\text { to } 10 \text { days) }\end{array}$ & $\begin{array}{l}\text { Diarrhoea; Nausea } \\
\text { and/or vomiting; } \\
\text { Fatigue }\end{array}$ \\
\hline
\end{tabular}

\section{Risk of bias in included studies}

Supplementary figure 1 presents the risk of bias assessment of the 16 included studies for each outcome. Overall and domain specific risk of bias judgments did not differ between the outcomes reported in each individual study, and most of the studies $(13 / 16,81.2 \%)$ presented significant methodological limitations.

\section{Adverse effects of the interventions (Table 2.)}

\section{Remdesivir}


Two studies ${ }^{19,33}$ including 1,281 patients reported on remdesivir specific adverse events. Both studies reported on acute kidney injury and one study ${ }^{19}$ including 1,048 patients reported on cognitive dysfunction/delirium. No studies reported on fatigue.

\section{Acute Kidney Injury}

Remdesivir may have little or no effect on acute kidney injury when compared to placebo (OR 0.85, 95\% CI: 0.51 to 1.41 ; RD 8 fewer per 1,000 participants, 95\% CI: 27 fewer to 21 more) (Supplementary figure 2.). The certainty of the evidence was low because of serious imprecision and serious indirectness (studies used change in serum creatinine rather than patient-important measures of acute kidney injury like renal replacement therapy requirement).

\section{Cognitive dysfunction/delirium}

Remdesivir may have little or no effect on cognitive dysfunction/delirium when compared to placebo (OR 1.22, 95\% CI: 0.48 to 3.11; RD 3 more per 1,000 participants, 95\% CI: 12 fewer to 19 more). The certainty of the evidence was low because of very serious imprecision.

\section{Hydroxychloroquine}

Ten studies ${ }^{21-26,29-32}$ including 3,663 patients reported on hydroxychloroquine specific adverse events. Seven studies including 3,287 patients reported cardiac toxicity, ${ }^{21,22,24,27,30-32}$ six trials including 979 patients reported diarrhoea, ${ }^{23-25,29-31}$ seven studies including 1,429 patients ${ }^{23-26,29-31}$ reported nausea and/or vomiting, one study ${ }^{30}$ including 423 patients reported on cognitive dysfunction/delirium and two studies ${ }^{24,31}$ including 180 patients reported on fatigue.

\section{Cardiac toxicity}

Definitions of cardiac toxicity varied between trials: RECOVERY defined the outcome as new major arrhythmias (supraventricular tachycardia, ventricular tachycardia or fibrillation or atrioventricular block requiring intervention), ${ }^{26}$ two studies as new arrhythmias, ${ }^{21,30}$ and one study as new arrhythmias or cardiac arrest. ${ }^{32}$ The remaining studies did not provide details about cardiac toxicity definition. Hydroxychloroquine may increase the risk of cardiac toxicity when compared to standard care or placebo (RD 10 more per 1,000 participants, $95 \%$ CI: 0 more to 30 
more) (Supplementary figure 3). The certainty of the evidence was low because of serious imprecision and risk of bias (unblinded studies with possible detection bias).

\section{Diarrhoea}

Hydroxychloroquine probably increases the risk of diarrhoea when compared to standard care or placebo (OR 1.95, 95\% CI: 1.40 to 2.73; RD 106 more per 1,000 participants, 95\% CI: 48 more to 175 more) (Supplementary figure 4). The certainty of the evidence was moderate because of imprecision as the optimal information size (OIS) was not met. Although most studies presented methodological limitations, we did not rate down for risk of bias (RoB) as our concerns were mitigated by a large effect size and indirect evidence showing consistent results. ${ }^{35}$

\section{Nausea and/or vomiting}

Hydroxychloroquine probably increases nausea and vomiting (OR 1.74, 95\% CI: 1.26 to 2.41; RD 62 more per 1,000 participants, 95\% CI: 23 more to 110 more) (Supplementary figure 5). The certainty of the evidence was moderate because of imprecision as OIS was not met. Although most studies presented methodological limitations, we did not rate down for RoB as our concerns were mitigated by a large effect size and indirect evidence showing consistent results. $^{35}$

\section{Cognitive dysfunction/delirium}

Hydroxychloroquine may increase cognitive dysfunction/delirium when compared to standard care or placebo (OR 1.59, 95\% CI: 0.77 to 3.28; RD 33 more per 1,000 participants, 95\% CI: 18 fewer to 84 more). The certainty of the evidence was low because of very serious imprecision.

\section{$\underline{\text { Fatigue }}$}

The effect of hydroxychloroquine on fatigue is uncertain when compared to standard care or placebo (OR 2.75, 95\% CI: 0.28 to 27.28 ; RD 82 more per 1,000 participants, 95\% CI: 38 fewer to 555 more) (Supplementary figure 6). The certainty of the evidence was very low because of very serious imprecision and serious risk of bias.

\section{Hydroxychloroquine with azithromycin}


Only one study ${ }^{21}$ including 667 patients reported drug-specific adverse effects for hydroxychloroquine with azithromycin. The study compared hydroxychloroquine with azithromycin, hydroxychloroquine alone, and standard care and reported on cardiac toxicity and nausea and/or vomiting. Other outcomes, including diarrhoea, cognitive dysfunction/delirium or fatigue were not reported.

\section{Cardiac toxicity}

The effect of hydroxychloroquine with azithromycin on cardiac toxicity is uncertain when compared to standard care or placebo (RD 10 more per 1,000 participants, 95\% CI: 10 fewer to 20 more), or hydroxychloroquine alone (RD 0 more per 1,000 participants, 95\% CI: 20 fewer to 20 more). The certainty of the evidence was very low because of very serious imprecision and serious risk of bias.

\section{Nausea and/or vomiting}

The effect of hydroxychloroquine with azithromycin on nausea and vomiting in uncertain when compared to standard care or placebo (OR 1.49, 95\% CI: 0.37 to 6.06 ; RD 8 more per 1,000 participants, 95\% CI: 11 fewer to 78 more), or hydroxychloroquine alone (OR 0.54, 95\% CI: 0.18 to 1.57 ; RD 20 fewer per 1,000 participants, 95\% CI: 37 fewer to 24 more). The certainty of the evidence was very low because of very serious imprecision and serious risk of bias.

\section{Lopinavir/ritonavir}

Four studies ${ }^{20,27,28,34}$ including 370 patients reported adverse effects of lopinavir/ritonavir. All four studies reported diarrhoea and nausea and/or vomiting. Two studies including 259 patients reported acute kidney injury ${ }^{20,27}$ and two studies including 254 patients reported fatigue. ${ }^{27,34}$ No studies reported on cognitive dysfunction/delirium.

\section{Acute Kidney Injury}

The effect of lopinavir/ritonavir on acute kidney injury is uncertain when compared to standard care or placebo (20 fewer per 1,000 participants, 95\% CI: 70 fewer to 20 more) (Supplementary figure 7). The certainty of the evidence was very low because of very serious imprecision and serious risk of bias. 


\section{Diarrhoea}

Lopinavir/ritonavir may increase the risk of diarrhoea when compared to standard care or placebo (OR 4.28, 95\% CI: 1.99 to 9.18; RD 168 more per 1,000 participants, 95\% CI: 58 more to 330 more) (Supplementary figure 8). The certainty of the evidence was low because of very serious imprecision. Although most studies presented methodological limitations, we did not rate down for RoB as our concerns were mitigated by a large effect size and indirect evidence showing consistent results. ${ }^{36}$

\section{Nausea and/or vomiting}

Lopinavir/ritonavir may increase the risk of nausea and/or vomiting when compared to standard care or placebo (RD 160 more per 1,000 participants, 95\% CI: 100 more to 210 more)

(Supplementary figure 9). The certainty of the evidence was low because of very serious imprecision. Although most studies presented methodologic limitations, we did not rate down for RoB as our concerns were mitigated by a large effect size and indirect evidence showing consistent results. ${ }^{36}$

\section{Fatigue}

The effect of lopinavir/ritonavir on fatigue is uncertain when compared to standard care or placebo (OR 1.56, 95\% CI: 0.53 to 4.58; 28 more per 1,000 participants, 95\% CI: 25 fewer to 154 more) (Supplementary figure 10). The certainty of the evidence was very low because of very serious imprecision and serious risk of bias.

Table 2. Summary of findings table

\begin{tabular}{|c|c|c|c|c|c|}
\hline \multirow{2}{*}{$\begin{array}{l}\text { Outcome } \\
\text { Timeframe }\end{array}$} & \multirow{2}{*}{$\begin{array}{l}\text { Study results and } \\
\text { measurements }\end{array}$} & Absolute e & & \multirow{2}{*}{$\begin{array}{l}\text { Certainty of the Evidence } \\
\text { (Quality of evidence) }\end{array}$} & \multirow{2}{*}{ Plain text summary } \\
\hline & & Standard care & Intervention & & \\
\hline
\end{tabular}


medRxiv preprint doi: https://doi.org/10.1101/2020.11.16.20232876; this version posted November 18, 2020. The copyright holder for this preprint (which was not certified by peer review) is the author/funder, who has granted medRxiv a license to display the preprint in perpetuity.

It is made available under a CC-BY-NC-ND 4.0 International license .

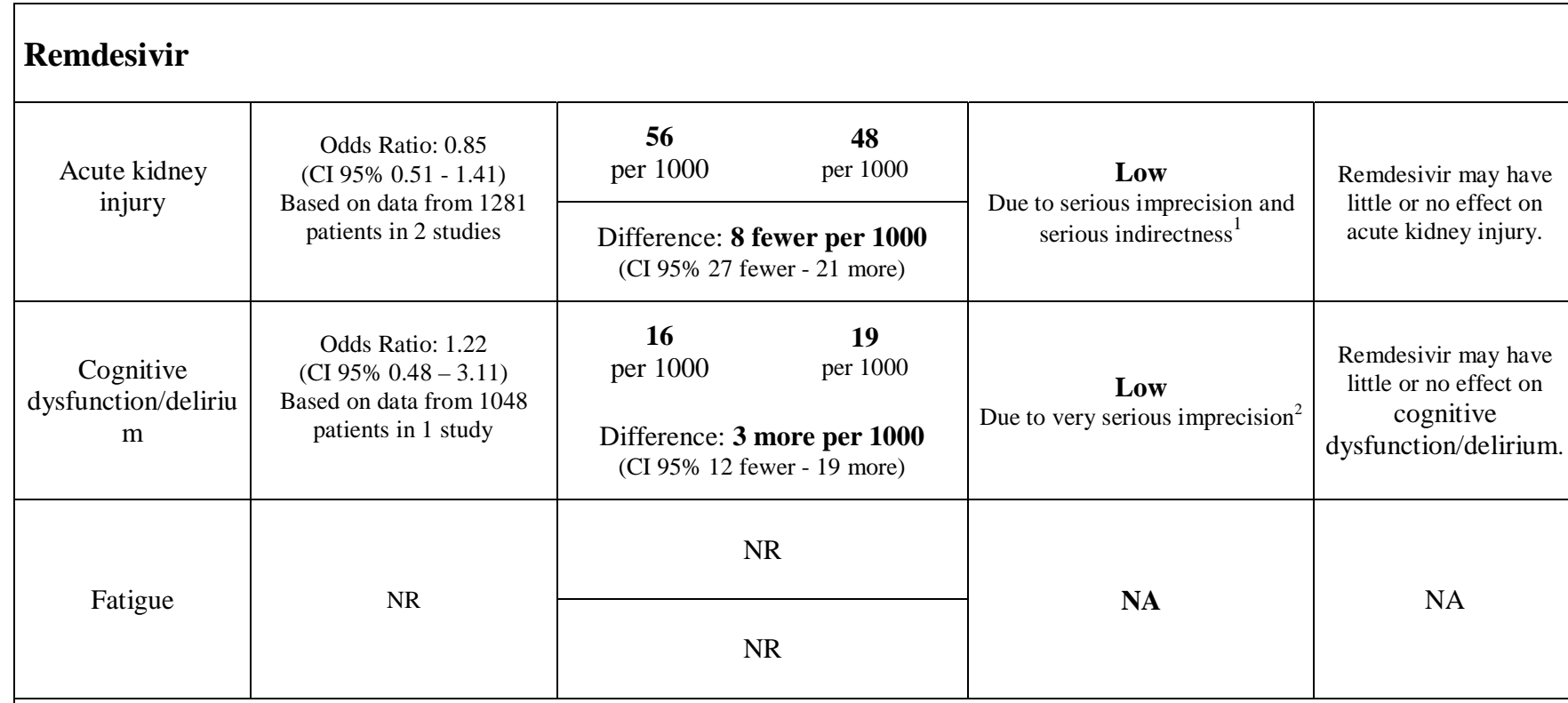

\section{Hydroxychloroquine}

\begin{tabular}{|c|c|c|c|c|}
\hline \multirow[t]{2}{*}{ Cardiac toxicity } & \multirow{2}{*}{$\begin{array}{l}\text { Based on data from } 3287 \\
\text { patients in } 7 \text { studies }\end{array}$} & $\begin{array}{c}\mathbf{4 6} \\
\text { per } 1000\end{array}$ & \multirow{2}{*}{$\begin{array}{c}\text { Low } \\
\begin{array}{c}\text { Due to serious imprecision and } \\
\text { risk of bias }\end{array}\end{array}$} & \multirow{2}{*}{$\begin{array}{l}\text { Hydroxychloroquine } \\
\text { may increase the risk of } \\
\text { cardiac toxicity, } \\
\text { including serious } \\
\text { arrhythmias. }\end{array}$} \\
\hline & & $\begin{array}{l}\text { Difference: } 10 \text { more per } 1000 \\
\quad(\text { CI } 95 \% 0 \text { more - } 30 \text { more })\end{array}$ & & \\
\hline Diarrhoea & $\begin{array}{c}\text { Odds Ratio: } 1.95 \\
(\mathrm{CI} 95 \% 1.40-2.73) \\
\text { Based on data from } 979 \\
\text { patients in } 6 \text { studies }\end{array}$ & $\begin{array}{cc}\mathbf{1 4 9} & \mathbf{2 5 5} \\
\text { per } 1000 & \text { per } 1000 \\
& \\
\text { Difference: } \mathbf{1 0 6} \text { more } \text { per } \mathbf{1 0 0 0} \\
\text { (CI 95\% } 48 \text { more } & \text { 175 more })\end{array}$ & $\begin{array}{c}\text { Moderate } \\
\text { Due to imprecision }\end{array}$ & $\begin{array}{l}\text { Hydroxychloroquine } \\
\text { probably increases the } \\
\text { risk of diarrhoea. }\end{array}$ \\
\hline $\begin{array}{l}\text { Nausea and/or } \\
\text { vomiting }\end{array}$ & $\begin{array}{c}\text { Odds Ratio: } 1.74 \\
\text { (CI 95\% } 1.26-2.41) \\
\text { Based on data from } 1429 \\
\text { patients in } 7 \text { studies }\end{array}$ & 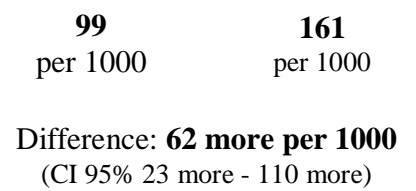 & $\begin{array}{c}\text { Moderate } \\
\text { Due to imprecision }^{4}\end{array}$ & $\begin{array}{l}\text { Hydroxychloroquine } \\
\text { probably increases the } \\
\text { risk of nausea and } \\
\text { vomiting. }\end{array}$ \\
\hline $\begin{array}{c}\text { Cognitive } \\
\text { dysfunction/deliriu } \\
\mathrm{m}\end{array}$ & $\begin{array}{c}\text { Odds Ratio: } 1.59 \\
\text { (CI 95\% } 0.77-3.28) \\
\text { Based on data from } 423 \\
\text { patients in } 1 \text { study }\end{array}$ & $\begin{array}{cc}\mathbf{6 2} & \mathbf{9 5} \\
\text { per } 1000 & \text { per } 1000 \\
& \\
\text { Difference: } & \mathbf{3 3} \text { more per } \mathbf{1 0 0 0} \\
\text { (CI 95\% } 18 \text { fewer }-84 \text { more) }\end{array}$ & $\begin{array}{c}\text { Low } \\
\text { Due to very serious imprecision }\end{array}$ & $\begin{array}{l}\text { Hydroxychloroquine } \\
\text { may increase } \\
\text { cognitive } \\
\text { dysfunction/delirium }\end{array}$ \\
\hline Fatigue & $\begin{array}{c}\text { Odds Ratio: } 2.75 \\
\text { (CI 95\% } 0.28-27.28) \\
\text { Based on data from } 180 \\
\text { patients in } 2 \text { studies }\end{array}$ & $\begin{array}{cc}\mathbf{5 4} & \mathbf{1 4} \\
\text { per } 1000^{5} & \text { per } 1000 \\
& \\
\text { Difference: } & \mathbf{8 2} \text { more per } \mathbf{1 0 0 0} \\
\text { (CI 95\% } 38 \text { fewer } & \text { 555 more) }\end{array}$ & $\begin{array}{l}\text { Very Low } \\
\text { Due to very serious imprecision } \\
\text { and serious risk of bias }{ }^{6}\end{array}$ & $\begin{array}{l}\text { The effect of } \\
\text { Hydroxychloroquine } \\
\text { on fatigue is } \\
\text { uncertain }\end{array}$ \\
\hline
\end{tabular}

\section{Hydroxychloroquine with azithromycin}

\begin{tabular}{|c|c|c|c|c|c|}
\hline Cardiac toxicity & $\begin{array}{c}\text { Based on data from } 667 \\
\text { patients in 1 study }\end{array}$ & $\begin{array}{c}\mathbf{6} \\
\text { per } 1000\end{array}$ & $\begin{array}{c}16 \\
\text { per } 1000\end{array}$ & $\begin{array}{c}\text { Very Low } \\
\text { Due to very serious imprecision }\end{array}$ & $\begin{array}{c}\text { The effect of } \\
\text { Hydroxychloroquine }\end{array}$ \\
\hline
\end{tabular}


medRxiv preprint doi: https://doi.org/10.1101/2020.11.16.20232876; this version posted November 18, 2020. The copyright holder for this preprint (which was not certified by peer review) is the author/funder, who has granted medRxiv a license to display the preprint in perpetuity.

It is made available under a CC-BY-NC-ND 4.0 International license .

\begin{tabular}{|c|c|c|c|c|c|}
\hline & & $\begin{array}{l}\text { Difference: } \\
\quad \text { (CI 95\% 10 }\end{array}$ & $\begin{array}{l}\text { e per } 1000 \\
-20 \text { more) }\end{array}$ & and serious risk of bias ${ }^{5}$ & $\begin{array}{l}\text { with azithromycin on } \\
\text { cardiac toxicity is } \\
\text { uncertain }\end{array}$ \\
\hline \multirow{2}{*}{$\begin{array}{l}\text { Nausea and/or } \\
\text { vomiting }\end{array}$} & \multirow{2}{*}{$\begin{array}{c}\text { Odds Ratio: } 1.49 \\
\text { (CI 95\% } 0.37-6.06) \\
\text { Based on data from } 667 \\
\text { patients in } 1 \text { study }\end{array}$} & $\begin{array}{c}\mathbf{1 7} \\
\text { per } 1000\end{array}$ & $\begin{array}{c}25 \\
\text { per } 1000\end{array}$ & \multirow{2}{*}{$\begin{array}{c}\text { Very Low } \\
\text { Due to very serious imprecision } \\
\text { and serious risk of bias }\end{array}$} & \multirow{2}{*}{$\begin{array}{l}\text { The effect of } \\
\text { Hydroxychloroquine } \\
\text { with azithromycin on } \\
\text { nausea and/or } \\
\text { vomiting is uncertain }\end{array}$} \\
\hline & & $\begin{array}{l}\text { Difference: } \\
\text { (CI 95\% } 11\end{array}$ & $\begin{array}{l}\text { e per } 1000 \\
-78 \text { more) }\end{array}$ & & \\
\hline \multirow{2}{*}{ Diarrhoea } & \multirow{2}{*}{ NR } & \multicolumn{2}{|c|}{ NR } & \multirow{2}{*}{ NA } & \multirow{2}{*}{ NA } \\
\hline & & & & & \\
\hline \multirow{2}{*}{$\begin{array}{c}\text { Cognitive } \\
\text { dysfunction/deliriu } \\
\mathrm{m}\end{array}$} & \multirow{2}{*}{ NR } & & & \multirow{2}{*}{ NA } & \multirow{2}{*}{ NA } \\
\hline & & & & & \\
\hline \multirow{2}{*}{ Fatigue } & \multirow{2}{*}{ NR } & & & \multirow{2}{*}{ NA } & \multirow{2}{*}{ NA } \\
\hline & & \multicolumn{2}{|c|}{ NR } & & \\
\hline
\end{tabular}

\section{Lopinavir/ritonavir}

\begin{tabular}{|c|c|c|c|c|}
\hline $\begin{array}{l}\text { Acute kidney } \\
\text { injury }\end{array}$ & $\begin{array}{c}\text { Based on data from } 259 \\
\text { patients in } 2 \text { studies }\end{array}$ & $\begin{array}{cc}\mathbf{4 5} & \mathbf{2 5} \\
\text { per } 1000 & \text { per } 1000 \\
& \\
\text { Difference: } \mathbf{2 0} \text { fewer per } \mathbf{1 0 0 0} \\
\text { (CI 95\% } 70 \text { fewer } & -20 \text { more })\end{array}$ & $\begin{array}{c}\text { Very Low } \\
\text { Due to very serious imprecision } \\
\text { and serious risk of bias }\end{array}$ & $\begin{array}{l}\text { The effect of } \\
\text { lopinavir/ritonavir on } \\
\text { acute kidney injury is } \\
\text { uncertain. }\end{array}$ \\
\hline Diarrhoea & $\begin{array}{c}\text { Odds Ratio: } 4.28 \\
\text { (CI 95\% } 1.99-9.18) \\
\text { Based on data from } 370 \\
\text { patients in } 4 \text { studies }\end{array}$ & $\begin{array}{cc}\mathbf{6 7} & \mathbf{2 3 5} \\
\text { per } 1000 & \text { per } 1000 \\
& \\
\text { Difference: } \mathbf{1 6 8} \text { more per } 1000 & (\text { CI } 95 \% 58 \text { more }-330 \text { more })\end{array}$ & $\begin{array}{c}\text { Low } \\
\text { Due to very serious imprecision }{ }^{7}\end{array}$ & $\begin{array}{l}\text { Lopinavir/ritonavir may } \\
\text { increase the risk of } \\
\text { diarrhoea. }\end{array}$ \\
\hline $\begin{array}{l}\text { Nausea and/or } \\
\text { vomiting }\end{array}$ & $\begin{array}{c}\text { Based on data from } 370 \\
\text { patients in } 4 \text { studies }\end{array}$ & 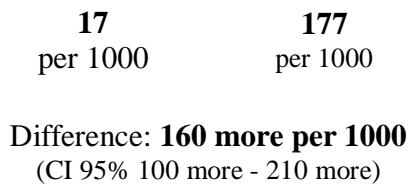 & $\begin{array}{c}\text { Low } \\
\text { Due to very serious imprecision }{ }^{7}\end{array}$ & $\begin{array}{l}\text { Lopinavir/ritonavir may } \\
\text { increase the risk of } \\
\text { nausea and vomiting. }\end{array}$ \\
\hline Fatigue & $\begin{array}{c}\text { Odds Ratio: } 1.56 \\
\text { (CI 95\% } 0.53-4.58) \\
\text { Based on data from } 254 \\
\text { patients in } 2 \text { studies }\end{array}$ & $\begin{array}{cc}\mathbf{5 4} & \mathbf{8 2} \\
\text { per 1000 } & \text { per 1000 } \\
\text { Difference: } & \mathbf{2 8} \text { more per 1000 } \\
\text { (CI 95\% 25 fewer } & \text { 154 more) }\end{array}$ & $\begin{array}{c}\text { Very Low } \\
\text { Due to very serious imprecision } \\
\text { and serious risk of bias }\end{array}$ & $\begin{array}{l}\text { The effect of } \\
\text { lopinavir/ritonavir on } \\
\text { fatigue is uncertain. }\end{array}$ \\
\hline $\begin{array}{c}\text { Cognitive } \\
\text { dysfunction/deliriu } \\
\mathrm{m}\end{array}$ & NR & NR & NA & NA \\
\hline
\end{tabular}


NR: Not reported; NA: Not applicable

1. Risk of bias: Not serious. We rated one trial as high risk of bias due to deviations from the intended intervention. Indirectness: Serious as studies used change in serum creatinine rather than patient-important measures of acute kidney injury.

2. Imprecision: Very serious. Confidence intervals include significant risk reduction and increase.

3. Risk of bias: Data primarily from unblinded studies, but we would expect that patients would be more closely monitored for cardiac toxicity in trials than in usual clinical practice. Therefore, we expect the risk of cardiac toxicity to be higher in usual clinical practice. Indirectness: Not serious. Trials measured cardiac toxicity differently in different trials. Imprecision: Serious. Confidence intervals include no effect.

4. Risk of bias: Serious. Most of the evidence is from unblinded trials, we didn't downgrade for RoB as our concerns were mitigated by a large effect size and indirect evidence showing consistent results. Imprecision: OIS not met.

5. As there were no events in the control arms of included studies, we used the baseline risk estimated for Lopinavir/ritonavir vs. SOC comparison for the same outcome.

6. Risk of bias: Serious. Most of the evidence is from unblinded trials. Imprecision: Very serious. Very small number of events.

7. Risk of bias: Serious. Most of the evidence is from unblinded trials; we did not downgrade for RoB as our concerns were mitigated by a large effect size and indirect evidence showing consistent results.; Imprecision: Very serious. Very small number of events.

\section{Sensitivity analyses}

Our interpretation of the results did not substantially change when using a Bayesian random effects model rather than frequentist fixed effects or when pooling relative estimates rather than absolute estimates (supplementary figures 11 to 22).

\section{Discussion}

This systematic review and meta-analysis - directly informing the living WHO guideline for COVID-19 therapeutics - provides a comprehensive overview of the evidence for drug-specific adverse effects of interest for three commonly used drugs for treatment of COVID-19. From 40 interventions included in our living network meta-analysis, ${ }^{2}$ we only included studies reporting on drug specific adverse events for remdesivir, hydroxychloroquine, hydroxychloroquine with azithromycin and lopinavir/ritonavir in this review as these drugs are receiving a high degree of interest. None of these interventions may increase the risk of adverse effects leading to discontinuation, however the certainty of the evidence was low for hydroxychloroquine and moderate for remdesivir, while no information was available for hydroxychloroquine with azithromycin, or lopinavir-ritonavir. ${ }^{2}$ In this review we found moderate certainty evidence that hydroxychloroquine increases the risk of diarrhoea and nausea and/or vomiting and low certainty evidence that it increases the risk of cardiac toxicity and cognitive dysfunction/delirium. For lopinavir/ritonavir we found low certainty evidence that it increases the risk of diarrhoea, and nausea and/or vomiting. Based on low or very low certainty evidence, we did not find evidence that remdesivir or lopinavir/ritonavir increase the risk of acute kidney injury or cognitive defunction/delirium.

\section{Strengths and limitations of this review}


The search strategy was comprehensive with explicit eligibility criteria, and no restrictions on language or publication status. To ensure expertise in all areas, the review team is composed of clinical and methods experts who have undergone training and calibration exercises for all stages of the review process. We assessed the certainty of the evidence using the GRADE approach and interpreted the results considering absolute, rather than relative, effects.

We evaluated only a limited number of adverse effects and interventions, as selected by the linked guideline panel. We included an adverse effect if any panel member believed it might be important to patients when deciding whether to use or not to use a drug. However, there may be other patient-important adverse drug effects that were not prespecified by the panel. Further, some may perceive that excluding surrogate outcomes, such as an increase in liver enzymes or electrocardiogram changes may lead to under-appreciation of potential harms, especially for surrogates that are more closely linked on the causal pathway to patient important harms.

So far there is limited evidence for the harms associated with most drugs as adverse effects were only reported by a limited number of studies. For comparisons with sufficient data, the primary limitation of the evidence was lack of blinding, which might introduce bias through differences in cointerventions or outcome assessment between randomisation groups. However, the large magnitude of effects observed resulted in moderate certainty that hydroxychloroquine causes specific adverse events.

These findings are consistent with "The Living Project" ( https://covid-nma.com/), which found an increase in any adverse events with hydroxychloroquine (RR 2.16, 95\% CI: 1.21 to 3.86) and lopinavir/ritonavir (RR 2.39, 95\% CI: 0.21 to 27.57), but not with remdesivir (RR 1.00, 95\% CI: 0.87 to 1.15$)$. However, they did not report on specific adverse events. Other systematic reviews found an increase in the risk of diarrhoea and nausea and/or vomiting with lopinavir-ritonavir ${ }^{37,38}$ and hydroxychloroquine, ${ }^{38-40}$ increase in arrhythmias and QTc interval prolongation with hydroxychloroquine alone, ${ }^{40-42}$ or combined with a macrolide, ${ }^{43,44}$ and no significant increase in renal failure with remdesivir. ${ }^{45}$

\section{Conclusion}


Hydroxychloroquine probably increases the risk of diarrhoea and nausea and/or vomiting and may increase the risk of cardiac toxicity and cognitive dysfunction/delirium. Remdesivir may have no effect on risk of acute kidney injury or cognitive dysfunction/delirium.

Lopinavir/ritonavir may increase the risk of diarrhoea and nausea and/or vomiting. These findings provide important information to support the development of evidence-based management strategies for patients with COVID-19.

\section{References}

1. John Hopkins University. Coronavirus Resource Center 2020 https://coronavirus.jhu.edu/map.html accessed November 102020

2. Siemieniuk RA, Bartoszko JJ, Ge L, Zeraatkar D, Izcovich A, Kum E, et al. Drug treatments for covid-19: living systematic review and network meta-analysis. BMJ. 2020 Jul 30;m2980.

3. Siemieniuk RA, Agoritsas T, Macdonald H, Guyatt GH, Brandt L, Vandvik PO. Introduction to BMJ Rapid Recommendations. BMJ. 2016 Sep 28;i5191.

4. Rochwerg B, Agarwal A, Zeng L, Leo Y-S, Appiah JA, Agoritsas T, et al. Remdesivir for severe covid-19: a clinical practice guideline. BMJ. 2020 Jul 30;m2924.

5. Lamontagne F, Agoritsas T, Macdonald H, Leo Y-S, Diaz J, Agarwal A, et al. A living WHO guideline on drugs for covid-19. BMJ. 2020 Sep 4;m3379.

6. Moher D, Liberati A, Tetzlaff J, Altman DG, for the PRISMA Group. Preferred reporting items for systematic reviews and meta-analyses: the PRISMA statement. BMJ. 2009 Jul 21;339(jul21 1):b2535-b2535.

7. World Health Organization. Global Research on coronavirus disease (COVID-19). https://www.who.int/emergencies/diseases/novel-coronavirus-2019/global-research-onnovel-coronavirus-2019-ncov

8. The Stephen B. Thacker CDC Library. COVID-19 Research Articles Downloadable Database: U.S. Centers for Disease Control and Prevention (CDC); 2020 https://www.cdc.gov/library/researchguides/2019novelcoronavirus/researcharticles.html [accessed 27 October 2020] 
9. Marshall IJ, Noel-Storr A, Kuiper J, Thomas J, Wallace BC. Machine learning for identifying Randomized Controlled Trials: An evaluation and practitioner's guide. Res Synth Methods 2018;9:602-14. PubMed doi:10.1002/jrsm.1287

10. Living evidence Repository for COVID-19. Epistemonikos Foundation. Available at: https://app.iloveevidence.com/loves/5e6fdb9669c00e4ac072701d [27 October 2020]

11. Norwegian Institute of Public Health. NIPH systematic and living map on COVID-19 evidence 2020 https://www.nornesk.no/forskningskart/NIPH_mainMap.html [accessed 27 October 2020.]

12. COVIDence systematic review software [program]. Melbourne, Australia: Veritas Health Innovation.

13. Sterne JAC, Savović J, Page MJ, et al. RoB 2: a revised tool for assessing risk of bias in randomised trials. BMJ 2019;366:14898. PubMed doi:10.1136/bmj.14898

14. Balduzzi S, Rücker G, Schwarzer G. How to perform a meta-analysis with R: a practical tutorial. Evid Based Cognitive Health. 2019 Nov;22(4):153-60.

15. GRADE Working Group . Grading quality of evidence and strength of recommendations. BMJ. 2004;328(7454):1490.

16. Hultcrantz M, Rind D, Akl EA, et al. The GRADE Working Group clarifies the construct of certainty of evidence. J Clin Epidemiol 2017;87:4-13. PubMed doi:10.1016/j.jclinepi.2017.05.006.

17. Rover C. Bayesian random-effects meta-analysis using the bayesmeta R package. Journal of Statistical Software 2017;93(5):1-51. doi: 10.18637/jss.v093.i06

18. Turner RM, Jackson D, Wei Y, et al. Predictive distributions for between-study heterogeneity and simple methods for their application in Bayesian meta-analysis. Stat Med 2015;34(6):984-98. doi: 10.1002/sim.6381 [published Online First: 2014/12/06]

19. Beigel JH, Tomashek KM, Dodd LE, Mehta AK, Zingman BS, Kalil AC, et al. Remdesivir for the Treatment of Covid-19 — Final Report. N Engl J Med. 2020 May 22;NEJMoa2007764.

20. Cao B, Wang Y, Wen D, et al. A Trial of Lopinavir-Ritonavir in Adults Hospitalized with Severe Covid-19. N Engl J Med 2020;382:1787-99. PubMed doi:10.1056/NEJMoa2001282 
21. Cavalcanti AB, Zampieri FG, Rosa RG, et al; Coalition Covid-19 Brazil I Investigators. Hydroxychloroquine with or without Azithromycin in Mild-to-Moderate Covid-19. N Engl J Med 2020. doi:10.1056/NEJMoa2019014

22. Chen Z, Hu J, Zhang Z, et al. Efficacy of hydroxychloroquine in patients with COVID19: results of a randomized clinical trial. medRxiv 2020. doi:10.1101/2020.03.22.20040758

23. Jun C, Liu D, Li L, et al. A preliminary study of hydroxychloroquine sulfate in patients with common 2019 coronavirus disease (COVID-19). Journal of Zhejiang University $2019 ; 49: 215-9$

24. Chen L, Zhang Z-y, Fu J-g, et al. Efficacy and safety of chloroquine or hydroxychloroquine in moderate type of COVID-19: a prospective open-label randomized controlled study. medRxiv 2020. doi:10.1101/2020.06.19.20136093

25. Chen C-P, Lin Y-C, Chen T-C, et al. A multicenter, randomized, open-label, controlled trial to evaluate the efficacy and tolerability of hydroxychloroquine and a retrospective study in adult patients with mild to moderate coronavirus disease 2019 (COVID-19). medRxiv 2020. doi:10.1101/2020.07.08.20148841

26. Horby P, Mafham M, Linsell L, et al. Effect of Hydroxychloroquine in Hospitalized Patients with COVID-19: Preliminary results from a multi-centre, randomized, controlled trial. medRxiv [Preprint] 2020. doi:10.1101/2020.07.15.20151852

27. Huang Y-Q, Tang S-Q, Xu X-L, et al. No statistically apparent difference in antiviral effectiveness observed among ribavirin plus interferon-alpha, lopinavir/ritonavir plus interferon-alpha, and ribavirin plus lopinavir/ritonavir plus interferon-alpha in patients with mild to moderate coronavirus disease 2019: results of a randomized, open-labeled prospective study. Front Pharmacol 2020;11:1071

28. Li Y, Xie Z, Lin W, et al. An exploratory randomized controlled study on the efficacy and safety of lopinavir/ritonavir or arbidol treating adult patients hospitalized with mild/moderate COVID-19 (ELACOI). medRxiv [Preprint] 2020.

29. Lyngbakken MN, Berdal J-E, Eskesen A, Kvale D, Olsen IC, Rueegg CS, et al. A pragmatic randomized controlled trial reports lack of efficacy of hydroxychloroquine on coronavirus disease 2019 viral kinetics. Nat Commun. 2020 Dec;11(1):5284. 
30. Skipper CP, Pastick KA, Engen NW, et al. Hydroxychloroquine in Nonhospitalized Adults With Early COVID-19: A Randomized Trial. Ann Intern Med 2020; published online 16 July. PubMed doi:10.7326/M20-4207

31. Tang W, Cao Z, Han M, et al. Hydroxychloroquine in patients with mainly mild to moderate coronavirus disease 2019: open label, randomised controlled trial. BMJ 2020;369:m1849. PubMed doi:10.1136/bmj.m1849

32. Ulrich RJ, Troxel AB, Carmody E, Eapen J, Bäcker M, DeHovitz JA, et al. Treating Covid-19 With Hydroxychloroquine (TEACH): A Multicenter, Double-Blind, Randomized Controlled Trial in Hospitalized Patients. Open Forum Infectious Diseases. 2020 Sep 23;ofaa446.

33. Wang Y, Zhang D, Du G, et al. Remdesivir in adults with severe COVID-19: a randomised, double-blind, placebo-controlled, multicentre trial. Lancet 2020;395:156978. PubMed doi:10.1016/S0140-6736(20)31022-9

34. Zheng F, Zhou Y, Zhou Z, et al. A Novel Protein Drug, Novaferon, as the Potential Antiviral Drug for COVID-19. medRxiv [Preprint] 2020. doi:10.1101/2020.04.24.20077735

35. Eljaaly K, Alireza KH, Alshehri S, Al-Tawfiq JA. Hydroxychloroquine safety: A metaanalysis of randomized controlled trials. Travel Medicine and Infectious Disease. 2020 Jul;36:101812.

36. Hill A, Balkin A. Risk factors for gastrointestinal adverse events in HIV treated and untreated patients. AIDS Rev. 2009 Mar;11(1):30-8.

37. Bhattacharyya A, Kumar S, Sarma P, Kaur H, Prajapat M, Shekhar N, et al. Safety and efficacy of lopinavir/ritonavir combination in COVID-19: A systematic review, metaanalysis, and meta-regression analysis. Indian J Pharmacol. 2020 Aug;52(4):313-23.

38. Liu W, Zhou P, Chen K, Ye Z, Liu F, Li X, et al. Efficacy and safety of antiviral treatment for COVID-19 from evidence in studies of SARS-CoV-2 and other acute viral infections: a systematic review and meta-analysis. CMAJ. 2020 Jul 6;192(27):E734-44.

39. Das RR, Jaiswal N, Dev N, Jaiswal N, Naik SS, Sankar J. Efficacy and Safety of Antimalarial Drugs (Chloroquine and Hydroxy-Chloroquine) in Treatment of COVID-19 Infection: A Systematic Review and Meta-Analysis. Front Med. 2020 Jul 29;7:482. 
40. Hernandez AV, Roman YM, Pasupuleti V, Barboza JJ, White CM. Hydroxychloroquine or Chloroquine for Treatment or Prophylaxis of COVID-19: A Living Systematic Review. Annals of Internal Medicine. 2020 Aug 18;173(4):287-96.

41. Shrestha DB, Budhathoki P, Khadka S, Rawal E. Hydroxychloroquine with or without macrolide and standard of care versus standard of care alone for COVID-19 cases: a systematic review and meta-analysis [Internet]. In Review; 2020 Jul [cited 2020 Nov 11]. Available from: https://www.researchsquare.com/article/rs-39092/v1

42. Jankelson L, Karam G, Becker ML, Chinitz LA, Tsai M-C. QT prolongation, torsades de pointes, and sudden death with short courses of chloroquine or hydroxychloroquine as used in COVID-19: A systematic review. Heart Rhythm. 2020 Sep;17(9):1472-9.

43. Kim MS, An MH, Kim WJ, Hwang T-H. Comparative efficacy and safety of pharmacological interventions for the treatment of COVID-19: A systematic review and network meta-analysis of confounder-adjusted 20212 hospitalized patients [Internet]. Infectious Diseases (except HIV/AIDS); 2020 Jun [cited 2020 Nov 11]. Available from: http://medrxiv.org/lookup/doi/10.1101/2020.06.15.20132407

44. Yang T-H, Chou C-Y, Yang Y-F, Chien C-S, Yarmishyn AA, Yang T-Y, et al. Systematic Review and Meta-analysis of the Effectiveness and Safety of Hydroxychloroquine in Treating COVID-19 Patients. Journal of the Chinese Medical Association [Internet]. 2020 Sep 15 [cited 2020 Nov 11];Publish Ahead of Print. Available from: https://journals.lww.com/10.1097/JCMA.0000000000000425

45. Piscoya A, Ng-Sueng LF, Parra del Riego A, Cerna-Viacava R, Pasupuleti V, Roman YM, et al. Efficacy and harms of remdesivir for the treatment of COVID-19: a systematic review and meta-analysis [Internet]. Infectious Diseases (except HIV/AIDS); 2020 May [cited 2020 Nov 11]. Available from: http://medrxiv.org/lookup/doi/10.1101/2020.05.26.20109595 